\title{
Mid-term Outcomes of Patients with Central Venous Occlusive Disease Undergoing Surveillance Venography and Intervention
}

Hui Lin Wong, ${ }^{1} M D, F R C R$, Shaun Xavier JM Chan, ${ }^{1} M B B S, F R C R$, FAMS, Satheesh Ramamuthy, ${ }^{2} M R C P C H, F R C R$, Kiang Hong Tay, ${ }^{1} M B B S, F R C R, F A M S$, Tze Tec Chong, ${ }^{3}$ MBBS, FACS, RPVI, Chieh Suai Tan, ${ }^{4}$ MBBS, MRCP, Ankur Patel, ${ }^{1} M B C h B, F R C R, F A M S$,

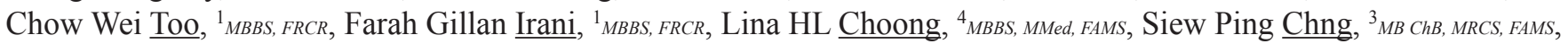
Bien Soo Tan, ${ }^{1}$ MBBS, FRCR, FAMS

\begin{abstract}
Introduction: To evaluate the mid-term outcomes of regular surveillance venography with or without percutaneous transluminal angioplasty in haemodialysis patients presenting with central venous occlusive disease. Materials and Methods: A single-centre retrospective analysis of haemodialysis patients who presented with central vein occlusion (CVO) and central vein stenosis (CVS) between January 2008 and December 2011 was performed. CVO and significant CVS were defined as $\mathbf{1 0 0 \%}$ and $>\mathbf{5 0} \%$ luminal narrowing respectively. Upon successful angioplasty on first presentation, patients were followed up with regular surveillance venography within 3-6 months of the intervention and were re-treated when a significant stenosis or occlusion was demonstrated. Data on patient's demographics, comorbidities, presenting symptoms, type of upper limb dialysis access, lesion characteristics and complications were collected. Technical success, primary patency and primary assisted patency were analysed. Results: Thirty-five patients with CVO and 77 patients with CVS were enrolled. The technical success of initial PTA was $\mathbf{7 7 \%}$ and $\mathbf{7 3 \%}$ for the CVO and CVS groups, respectively. The primary patency at 3 months was $65 \%$ and $55 \%$ for the CVO group and CVS group, respectively $(P=\mathbf{0 . 3 2})$. The primary assisted patency at 1 year was $88 \%$ and $99 \%$ for the CVO group and CVS group, respectively $(P=0.009)$. At 2 years, the primary assisted patency were $77 \%$ and $90 \%$, respectively $(P=0.07)$. There was significant difference in the overall primary assisted patency $(P=0.048)$ between the CVO and CVS groups. Conclusion: CVOs are more difficult to treat than CVS. High primary assisted patency rates can be achieved with surveillance venography, albeit at the expense of increased number of interventions. Further cost effectiveness studies need to be performed to study the true benefit of our surveillance programme.
\end{abstract}

Ann Acad Med Singapore 2020;49:360-66

Key words: Dialysis circuit, End stage renal failure, Renal replacement therapy

\section{Introduction}

Central venous occlusive disease (CVD) is a common problem seen in end-stage renal failure (ESRF) patients on haemodialysis, occurring in $29 \%-40 \%$ of patients. ${ }^{1}$ The National Kidney Foundation-Kidney Disease Outcomes Quality Initiative (NKF-KDOQI) recommends percutaneous transluminal angioplasty (PTA) as the first line treatment for CVD. For recurrent stenoses (within 3 months) or stenoses refractory to PTA, the recommended treatment is stenting. ${ }^{2}$
The treatment of central vein occlusion (CVO) is technically more challenging than that of central vein stenosis (CVS). At our institution, a strategy of 3-6 monthly surveillance venography was instituted for haemodialysis patients with CVD. Significant stenoses ( $>50 \%$ luminal loss) were initially treated with PTA. If these lesions were found to be again significant on follow-up surveillance venography, regardless of symptoms, they were re-treated to prevent subsequent progression to CVO.

\footnotetext{
${ }^{1}$ Department of Vascular and Interventional Radiology, Singapore General Hospital, Singapore

${ }^{2}$ Department of Radiology, Apollo Hospital, Chennai, India

${ }^{3}$ Department of Vascular Surgery, Singapore General Hospital, Singapore

${ }^{4}$ Department of Renal Medicine, Singapore General Hospital, Singapore

Address for Correspondence: Dr Wong Hui Lin, Department of Vascular and Interventional Radiology, Singapore General Hospital, Outram Road, Singapore 169608

Email address: huilin.wong@mohh.com.sg
} 
This single arm study evaluates the mid-term outcomes of patients with CVO and CVS who underwent regular surveillance venography after successful PTA on initial presentation.

\section{Material and Methods}

This is a retrospective single-centre study analysing consecutive cases of central vein PTA performed at our institution between January 2008 and December 2011. Approval was obtained from our Institutional Review Board for access to these patients' medical records. Patients' demographics, comorbidities, presenting symptoms, indications for central vein venogram, details of each PTA or venogram, location of haemodialysis access, the use of tunnelled central venous catheter (CVC), the use of antiplatelet or anticoagulant medication and procedural-related complications were recorded. The venograms were reviewed by 2 radiologists together in consensus. If the patient had presented with more than one central vein lesion, only the more severe lesion was studied.

\section{Patients}

ESRF patients on arteriovenous (AV) haemodialysis who newly presented with CVO and CVS (ipsilateral central veins or superior vena cava) between January 2008 and December 2011 were recruited into the study. Inclusion criteria were patients with ipsilateral functioning AV haemodialysis access on the upper extremities and who had not undergone PTA of central veins prior to 2008. These patients were included if the lesion could be crossed and angioplastied on the first procedure. These patients were then placed on regular surveillance venography (within 3-6 months of the initial PTA) and they were followed up for a period of 2 years. Exclusion criteria were patients with only contralateral CVS or CVO, lesions which could not be treated percutaneously at presentation, patients who had undergone stenting on their first intervention or those who had no follow-up after the first PTA.

\section{Definitions}

Central veins are defined as the subclavian vein, brachiocephalic vein or the superior vena cava. Regular surveillance venography (with and without subsequent PTA) was defined as that performed within 3-6 months after initial venography and PTA. The endpoints of follow-up included cessation of haemodialysis due to peritoneal dialysis or renal transplantation, creation of a new AV access on the contralateral arm or in either groin or permanent occlusion, stenting of the central vein lesion or patients lost to follow-up or death. Mid-term outcome is defined as 2 years from the initial PTA.

An angiographic significant central lesion is defined as more than $50 \%$ luminal loss. Technical success was defined as less than 30\% residual stenosis after PTA. Primary patency of the central veins was defined as the time interval between the initial and next intervention. Primary assisted patency of the central vein was defined as the interval between the initial intervention and the date of permanent occlusion or stenting of the central vein lesion. Complications were classified in accordance with the Society of Interventional Radiology criteria. ${ }^{3}$

\section{Technique}

An initial central venogram was performed via a $23 \mathrm{G}$ cannula inserted into the upper limb fistula to document the location and degree of CVD. PTA would be attempted in the presence of a significant CVS or CVO. After gaining venous access via the appropriate upper limb or common femoral vein, a 4-Fr diagnostic catheter (Berenstein, Cordis, Warren, New Jersey, USA) and an 0.018-0.035-in hydrophilic guidewire (Terumo, Tokyo, Japan) were introduced to cross the lesion. PTA of the lesion was performed using standard techniques with an appropriately sized PTA balloon which was inflated until the lesion was effaced or up to the rated burst pressure, whichever was lower. The balloons used were sized to the adjacent normal venous segment and they ranged from 12 to $16 \mathrm{~mm}$ and were of $40 \mathrm{~mm}$ length (Powerflex, Cordis, Warren, New Jersey, USA; Atlas, Bard Peripheral Vascular, Tempe, Arizona, USA).

In chronic occlusions where antegrade or retrograde access alone was inadequate for PTA, a combined antegrade-retrograde approach was employed. In the latter technique, vascular sheaths were inserted antegradely into the upper arm dialysis circuit and in the appropriate common femoral vein. A suitable guide wire was first used to cross the CVO from either direction and exteriorised via the opposite sheath with the aid of a snare. Once through and through access was achieved, a small balloon was used for pre-dilatation. After which, PTA was performed with an optimally sized balloon. Prolonged inflations of up to 3 minutes were typically performed. A completion venogram was then procured to document the result post-PTA. 


\section{Statistical Analysis}

Statistical analyses were performed using SPSS Version. 21. The data was reported as mean \pm standard deviation or count (percentages). Primary and primary assisted patency rate of central veins was determined using Kaplan-Meier analysis and log rank test was used to compare patency rates between the 2 groups. Cox regression was performed to identify predictive factors that were associated with the central vein patency rates. Chi-square test and independent sample Student's t-test were used to compare categorical and continuous variables between the groups. The level of significance was set at 0.05 .

\section{Results}

A total of 176 patients on haemodialysis presented with CVD during the 4-year study period, of which 52 patients had CVO and 124 patients had CVS. Among these patients, 7 patients had lesions that could not be traversed by a guidewire and PTA was not performed, 4 patients underwent stenting on their first intervention and 53 patients were lost to follow-up after initial PTA. Of the remaining 112 patients, 35 were in the CVO group and 77 in the CVS group.

The mean duration of follow-up was $16 \pm 7.2$ months for the CVO group and $16 \pm 7.7$ months for the CVS group. The mean number of PTA per patient in the first year was $3.7 \pm 1.3$ and $3.3 \pm 1.1$ for the CVO group and CVS group, respectively. The mean number of PTA over the 2-year follow-up period was $5.8 \pm 2.7$ and $5.0 \pm 2.6$ for the CVO group and CVS group, respectively.

The demographics and co-morbidities of both groups are summarised in Table 1 . The 2 groups were not significantly different in terms of co-morbidities but there were significantly more male patients $(P=0.03)$ in the CVS group.

Table 1. Patients' Demographics and Risk Factors (Reported as N (\%) for Categorical Variables and Mean \pm SD for Continuous Variables)

\begin{tabular}{|c|c|c|c|}
\hline & $\begin{array}{c}\text { CVO } \\
(\mathbf{N}=35)\end{array}$ & $\begin{array}{c}\text { CVS } \\
(\mathbf{N}=77)\end{array}$ & $P$ value \\
\hline \multicolumn{4}{|l|}{ Demographics } \\
\hline Male & $13(37 \%)$ & $46(60 \%)$ & 0.03 \\
\hline Female & $22(63 \%)$ & $31(40 \%)$ & 0.03 \\
\hline Patient age (years) & $63 \pm 9.3$ & $63 \pm 9.8$ & 0.46 \\
\hline Follow-up (months) & $16 \pm 7.2$ & $16 \pm 7.7$ & 0.45 \\
\hline Number of PTA over 2 years & $5.8 \pm 2.7$ & $5.0 \pm 2.6$ & 0.46 \\
\hline Number of PTA in $1^{\text {st }}$ year & $3.7 \pm 1.3$ & $3.3 \pm 1.1$ & 0.63 \\
\hline \multicolumn{4}{|l|}{ Comorbidities } \\
\hline Diabetes & $20(57 \%)$ & $41(53 \%)$ & 0.70 \\
\hline Hypertension & $33(94 \%)$ & $76(99 \%)$ & 0.23 \\
\hline Coronary artery disease & $14(40 \%)$ & $36(47 \%)$ & 0.51 \\
\hline Hyperlipidemia & $32(91 \%)$ & $62(81 \%)$ & 0.15 \\
\hline History of smoking* & $2(7 \%)$ & $7(11 \%)$ & 0.72 \\
\hline \multicolumn{4}{|l|}{ Access location } \\
\hline Upper arm & $28(80 \%)$ & $67(87 \%)$ & 0.34 \\
\hline \multicolumn{4}{|l|}{ Side of CVS/CVO } \\
\hline Left & $24(69 \%)$ & $41(53 \%)$ & 0.22 \\
\hline Right & $11(31 \%)$ & $32(42 \%)$ & 0.22 \\
\hline Use of anti-platelets or anticoagulants & $10(29 \%)$ & $31(40 \%)$ & 0.23 \\
\hline History of tunnelled CVC insertion* & $31(88 \%)$ & $61(89 \%)$ & 0.86 \\
\hline
\end{tabular}

* $\%$ computed based on available data 
The most common indication for initial referral for PTA was swelling of the upper extremity (CVO, $43 \%$; CVS, $48 \%, P>0.05)$ in both groups. The other indications and distribution of the central vein lesions are shown in Table 2. For both groups, the brachiocephalic vein (CVO, 97\%; CVS, 68\%, $P=0.001)$ was the most common site.

\section{Outcome}

The technical success rate of initial PTA was $77 \%$ for the CVO group and $73 \%$ for the CVS group $(P=0.6)$. We were only interested in determining the outcomes of patients who underwent our surveillance programme after successful initial PTA, so the 7 patients who had lesions that could not be crossed on initial presentation were excluded.

The 3-month primary patency of the central veins was $65 \%$ and $55 \%$ for the CVO and CVS group, respectively $(P=0.32)$ and the 6 -month primary patency was $6 \%$ for both groups $(P=1.0)$.

The 6-month primary assisted patency of the central vein was $91 \%$ and $99 \%$ for the CVO and CVS groups, respectively $(P=0.03)$; the 1 -year primary assisted patency was $88 \%$ and $99 \%$ for the CVO and CVS groups, respectively $(P=0.009)$. The 2 -year primary assisted patency was $77 \%$ and $90 \%$, respectively $(P=0.07)$. Log rank test showed significant difference in the overall primary assisted patency between the

Table 2. Indication for Referral for Initial PTA and Distribution of Central Vein Lesions (Reported as N(\%))

\begin{tabular}{|c|c|c|c|}
\hline & $\begin{array}{c}\text { CVO } \\
\mathrm{N}=\mathbf{3 5}\end{array}$ & $\begin{array}{c}\text { CVS } \\
\mathbf{N}=77\end{array}$ & $P$ value \\
\hline \multicolumn{4}{|l|}{ Indication } \\
\hline Swelling & $15(43 \%)$ & $37(48 \%)$ & 0.61 \\
\hline \multicolumn{4}{|l|}{ Malfunction access } \\
\hline High venous pressure & $2(6 \%)$ & $9(12 \%)$ & 0.50 \\
\hline Poor access flow & $8(23 \%)$ & $12(16 \%)$ & 0.35 \\
\hline Bleeding post-dialysis & $0(0 \%)$ & $1(1 \%)$ & 1.00 \\
\hline Thrombosis & $2(6 \%)$ & $4(5 \%)$ & 1.00 \\
\hline Not specified & $4(11 \%)$ & $6(8 \%)$ & 0.50 \\
\hline Asymptomatic & $4(11 \%)$ & $8(10 \%)$ & 1.00 \\
\hline \multicolumn{4}{|l|}{ Distribution of lesions } \\
\hline Subclavian vein & $1(3 \%)$ & $21(27 \%)$ & 0.03 \\
\hline Brachiocephalic vein & $34(97 \%)$ & $52(68 \%)$ & 0.001 \\
\hline Superior vena cava & $0(0 \%)$ & $4(5 \%)$ & 0.31 \\
\hline
\end{tabular}

2 groups $(P=0.048)$. This data is summarised in Table 3 and Figures $1 \mathrm{~A}$ and 1B.

Table 3. Primary and Primary Assisted Patency of Central Vein

\begin{tabular}{lccc}
\hline & CVO & CVS & P value \\
\hline Primary patency & & & 0.45 \\
3 months & $65 \%$ & $55 \%$ & 0.32 \\
6 months & $6 \%$ & $6 \%$ & 1.0 \\
Primary assisted patency & & & 0.048 \\
12 months & $88 \%$ & $99 \%$ & 0.009 \\
24 months & $77 \%$ & $90 \%$ & 0.07
\end{tabular}

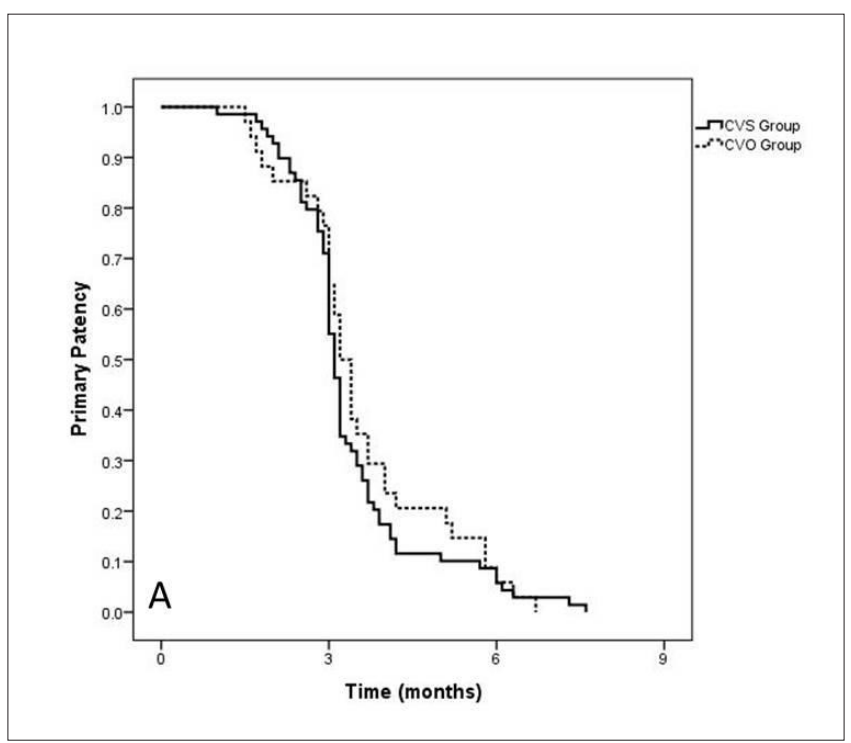

Fig. 1A. Primary patency of central veins.

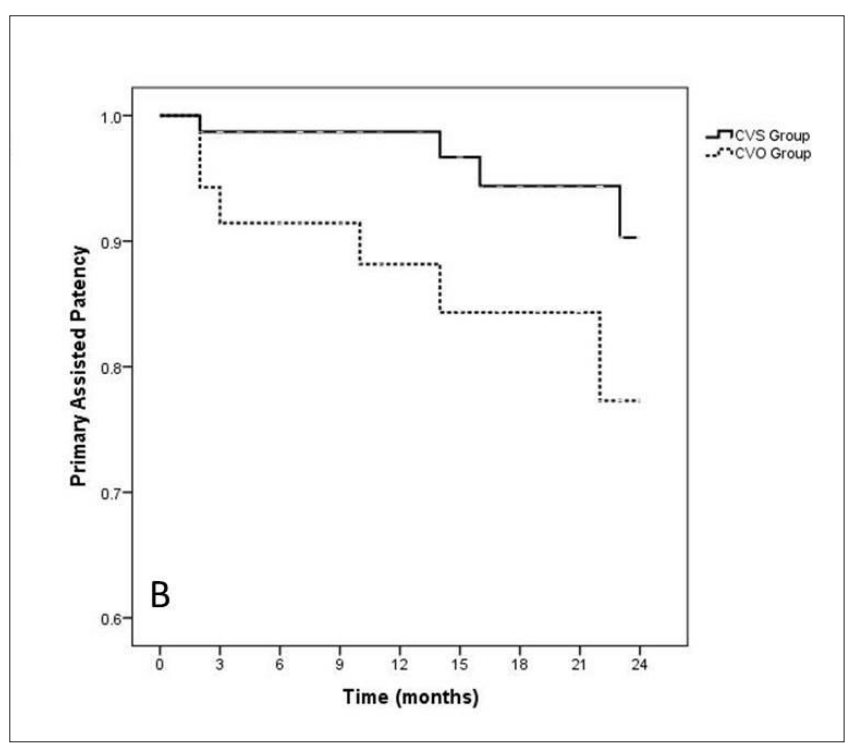

Fig. 1B. Primary assisted patency of central veins. 
Multivariable analysis was performed on the following factors: age, gender, co-morbidities, history of tunnelled CVC use, location of AV haemodialysis access, side of lesion, and use of antiplatelet/ anticoagulation medication did not yield any significant association in relation to the primary assisted patency.

The complication rates for both the CVO and CVS groups were low, with $1 \%$ or less directly related to PTA of the central veins. These complications were minor and, in all instances, managed conservatively. A total of 8 patients died within 30 days of the procedure, none of which were directly related to PTA. Three patients died from diabetic wound related sepsis, 3 from sequalae of their chronic renal disease, 1 from a cerebrovascular event and 1 from an acute cardiac event (Table 4).

At the end of the study period, 12 (34\%) patients in the CVO group and 39 (51\%) patients in the CVS group were on follow-up. Five (14\%) patients in the CVO group and $3(4 \%)$ patients in the CVS group underwent stenting of the central vein lesion or had permanent CVO. There were $4(11 \%)$ patients and $11(14 \%)$ patients in the CVO and CVS groups, respectively, who were lost to follow-up.

Table 4. Complications (Reported as N)

\begin{tabular}{|c|c|c|}
\hline & $\begin{array}{c}\text { CVO } \\
\text { (Total no. of } \\
\text { PTA = 202) }\end{array}$ & $\begin{array}{c}\text { CVS } \\
\text { (Total no. of } \\
\text { PTA = 387) }\end{array}$ \\
\hline \multicolumn{3}{|l|}{ Minor Complications } \\
\hline \multicolumn{3}{|l|}{$\begin{array}{l}\text { Directly related to PTA } \\
\text { of central vein }\end{array}$} \\
\hline Contrast extravasation & 1 & \\
\hline Haematoma & 1 & 2 \\
\hline Ruptured PTA balloon & & 1 \\
\hline \multicolumn{3}{|l|}{$\begin{array}{l}\text { Not directly related to PTA } \\
\text { of central veins }\end{array}$} \\
\hline Inadvertent embolisation & 1 & \\
\hline Contrast extravasation & 1 & 6 \\
\hline Post angioplasty thrombosis & & 1 \\
\hline Dissection of vein & & 1 \\
\hline Ruptured vein & & 1 \\
\hline 30-Day Mortality & 2 & 6 \\
\hline
\end{tabular}

\section{Discussion}

While CVD is a common problem that affects haemodialysis patients, the true prevalence of $\mathrm{CVO}$ is not known. The presence of CVO, if permanent, usually precludes the subsequent creation of new dialysis access on the ipsilateral upper limb. ${ }^{4}$

Current guidelines recommend stenting of CVD lesions for recurrent stenoses or stenoses that are refractory to PTA. ${ }^{2}$ In our institution, stenting is less preferred as it can impede future endovascular and surgical treatment options when major venous junctions are stented. Unlike malignant CVS and CVO where there is consensus for stenting, the management of benign CVS and CVO, as in our study population, is still being debated. ${ }^{5}$ In selected patients with permanent $\mathrm{CVO}$ and who have no other access site, the use of Haemodialysis Reliable Outflow (HeRO) graft (Merit Medical, Utah, USA) can serve as an alternative vascular access, circumventing the CVO. ${ }^{6}$ However, this is considered a relatively new approach in the local setting. In view of the challenges related to CVO, we embarked on regular surveillance venography for our patients with CVD to prevent subsequent progression to CVO.

It is estimated that $50 \%$ of patients with significant CVD develop clinical symptoms. ${ }^{7}$ This is similar to our study population where $48 \%$ of the patients with CVS and $43 \%$ of the patients with CVO were symptomatic at first presentation. The remainder of the patients presented with malfunctioning dialysis access.

Our initial PTA technical success rates were $77 \%$ and $73 \%$ in the CVO and CVS groups, respectively, comparable with the literature which ranged from $70-90 \% .^{8-10}$ Of note, there were 7 patients with CVO lesions which could not be crossed at the initial PTA. If these 7 patients were included in the calculation, the initial technical success rate for PTA in patients who first presented with $\mathrm{CVO}$, prior to entry into our surveillance programme, would have been significantly lower at $64 \%$. In other words, CVO is technically more challenging to treat than CVS.

The primary patency of the central veins after PTA has been reported in the literature to be $49-63 \%$ and $29-55 \%$ at 3 and 6 months, respectively., ${ }^{9,1-13}$ In our study, the primary patency for the CVO group was $65 \%$ and $6 \%$ at 3 and 6 months, respectively. The primary patency for the CVS group was $55 \%$ and $6 \%$ at 3 and 6 months, respectively. The fact that most of 
our patients maintain primary patency at 3 months validated our strategy of not surveying them earlier than 3 months. Of note, our low primary patency rate at 6 months was due to our institutional practice of intervening as a result of our surveillance programme.

In a small study of 24 venograms with CVS, it has been shown that PTA was associated with rapid restenosis progression. ${ }^{14}$ In another retrospective study, withholding PTA in asymptomatic patients who had CVS resulted in significantly higher central vein patency rates compared with symptomatic patients who were treated. The primary patency rate at 36 months for the asymptomatic and treated symptomatic groups were $67 \%$ and $18 \%$, respectively. ${ }^{10}$ These studies suggested that asymptomatic patients with CVS should not be treated. We recognise that restenosis is an inevitable outcome for most patients who have been treated with PTA, with endothelial proliferation as the main cause of restenosis. ${ }^{8}$ However, the subject numbers are much smaller compared to our study and CVO was not addressed separately.

Several studies in the literature have reported their 1 -year and 2-year primary assisted patency to be $73-82 \%$ and $56-62 \%$, respectively (Table 5). ${ }^{9,15-16}$ We achieved a higher primary assisted patency rate of $88-99 \%$ at 1 -year and $77-90 \%$ at 2 -years. In 2 comparable studies, the mean number of PTA per patient was $1.5 \pm 1.0$ and $2.0 \pm 1.6$, respectively, over a mean follow-up period of 1.8-3.3 years. ${ }^{9,15}$ In our study, the mean number of PTA per patient was $5.8 \pm 2.7$ for the CVO group and $5.0 \pm 2.6$ for the CVS group over a mean follow-up period of 1.3 years. With our surveillance programme, we are therefore able to prolong patency of the central veins, albeit at the expense of an increased number of interventions.

In our study, the overall primary assisted patency of the central veins for the CVO and CVS groups were significantly different (overall, $P=0.048$ ). This indicates that patients who initially presented with CVO deteriorated faster despite being on regular follow-up. Fourteen percent of our patients in the CVO group and $4 \%$ in the CVS group became permanently occluded or required stenting. In a study of 9 patients with CVO, Kim YC et al reported no significant difference in the primary assisted patency post-PTA between $\mathrm{CVO}$ and CVS. ${ }^{15}$ In another study reporting the use of stent grafts in CVD, while not directly comparable, a significantly shorter primary patency was also found in patients with CVO than with CVS. ${ }^{17}$

There are several limitations in our study. Firstly, initial patient referral was based on individual physician's criteria. Secondly, the PTA technique among operators was not standardised. Thirdly, the measurable dialysis information was also not available. Further, the number of patients who were symptomatic during subsequent follow-up was not completely available as this was not consistently recorded. Lastly, the severity of the central vein lesions was determined only via imaging and no haemodynamic measurements were made. On the other hand, the major strengths of our study include a larger population size and CVO group which facilitated statistical analysis.

In conclusion, CVD remains a difficult problem in ESRF patients on haemodialysis and should be managed in a multidisciplinary manner. We have shown that high primary assisted patency rates can be achieved with an increased number of interventions. As CVO is harder to treat than CVS, pre-emptive PTA of CVS to prevent progression to CVO will preserve central vein patency. This will allow the ipsilateral upper limb to be used for dialysis access. Further cost-effectiveness studies should be performed to determine the true benefit of our strategy of surveillance venography for CVD.

Table 5. Summary of Assisted Primary Patency in Studies of Central Vein PTA

\begin{tabular}{|c|c|c|c|c|c|c|}
\hline \multirow[t]{2}{*}{ Study } & \multirow[t]{2}{*}{ Year } & \multirow[t]{2}{*}{$\mathbf{N}$} & \multirow[t]{2}{*}{ Initial success $(\%)$} & \multicolumn{3}{|c|}{ Primary assisted patency $(\%)$ at } \\
\hline & & & & 6 months & 12 months & 24 months \\
\hline Our study - CVS Group & - & 77 & 73 & 99 & 99 & 90 \\
\hline Bakken et $\mathrm{al}^{9}$ & 2007 & 47 & 82 & 77 & 73 & 57 \\
\hline Kim et $\mathrm{al}^{15}$ & 2009 & 26 & - & 100 & 78 & 56 \\
\hline Ozyer et $\mathrm{al}^{16}$ & 2009 & 94 & - & 92 & 82 & 62 \\
\hline
\end{tabular}




\section{REFERENCES}

1. Lumsden AB, MacDonald MJ, Isiklar H, Martin LG, Kikeri D, Harker LA, et al. Central venous stenosis in the hemodialysis patient: incidence and efficacy of endovascular treatment. Cardiovasc Surg 1997;5:504-9.

2. National Kidney Foundation. Guideline 20.K/DOQI Clinical Practice Guidelines for Vascular Access: update 2000. Am J Kidney Dis 2001;37:S137-81

3. Gary RJ, Sacks D, Martin LG, Trerotola SO, Society of Interventional Radiology Technology Assessment Committee. Reporting standards for percutaneous interventions in dialysis access. J Vasc Interv Radiol 2003; 14:S433-42.

4. AgarwalAK. Central vein stenosis. Am J Kidney Dis 2013 ;61:1001-15.

5. Tan BS, Htoo MM, Yeong KY. The use of metallic stents in the treatment of malignant superior vena caval obstruction. Ann Acad Med Singapore 1995;24:198-203

6. Koh K, Koh YX, Choke ET, Wang JC, Kian CJ. Alternative strategies for central venous stenosis and occlusion in patients requiring haemodialysis access. Ann Acad Med Singapore 2017;46:39-41.

7. Schwab SJ, Quarles LD, Middleton JP, Cohan RH, Saeed M, Dennis VW. Hemodialysis-associated subclavian vein stenosis. Kidney Int $1998 ; 33: 1156-9$.

8. Kovalik EC, Newman GE, Suhocki P, Knelson M, Schwab SJ. Correction of central venous stenoses: use of angioplasty and vascular Wallstents. Kidney Int 1994 ;45:1177-81.

9. Bakken AM, Protack CD, Saad WE, Lee DE, Waldman DL, Davies MG. Long-term outcomes of primary angioplasty and primary stenting of central venous stenosis in hemodialysis patients. J Vasc Surg 2007;45:776-83.
10. Renaud CJ, Francois M, Nony A, Fodil-Cherif M, TurmelRodrigues L. Comparative outcomes of treated symptomatic versus non-treated asymptomatic high-grade central vein stenoses in the outflow of predominantly dialysis fistulas. Nephrol Dial Transplant 2012; 27:1631-8.

11. Dammers R, de Haan MW, Planken NR, Van Der Sande FM, Tordoir JH. Central vein obstruction in hemodialysis patients: results of radiological and surgical intervention. Eur J Vasc Endovasc Surg 2003;26:317-21.

12. Surowiec SM, Fegley AJ, Tanski WJ, Sivamurthy N, Illig KA, Lee DE, et al. Endovascular management of central venous stenoses in the hemodialysis patient: results of percutaneous therapy. Vasc Endovascular Surg 2004;38:349-54.

13. Beathard GA. Percutaneous transvenous angioplasty in the treatment of vascular access stenosis. Kidney Int 1992;42:1390-7.

14. Levit RD, Cohen RM, Kwak A, Shlansky-Goldberg RD, Clark TW, Patel AA, et al. Asymptomatic central venous stenosis in hemodialysis patients. Radiology 2006 ;238:1051-6.

15. Kim YC, Won JY, Choi SY, Ko HK, Lee KH, Lee DY, et al. Percutaneous treatment of central venous stenosis in hemodialysis patients:long-term outcomes. Cardiovasc Intervent Radiol 2009;32:271-8.

16. Ozyer U, Harman A, Yildirim E, Aytekin C, Karakayali F, Boyvat F. Long-term results of angioplasty and stent placement for treatment of central venous obstruction in 126 hemodialysis patients: a 10-year single-center experience. AJR Am J Roentgenol 2009; 193:1672-9

17. Jones RG, Willis AP, Jones C, McCafferty IJ, Riley PL. Long-term results of stent-graft placement to treat central venous stenosis and occlusion in hemodialysis patients with arteriovenous fistulas. J Vasc Interv Radiol 2011;22:1240-5. 